\title{
Apport des retours d'expérience pour la mise en sécurité dans les mines de fer de Lorraine
}

J.-P. JOSIEN

Expert minier

Ancien Directeur

de Géodéris

63, rue Pierre-Sémard 54000 Nancy

jeanpierre.josien@yahoo.fr

\section{J.-P. PIGUET}

École des Mines de Nancy Parc de Saurupt CS 14234 54042 Nancy Cedex

jack-pierre.piguet @mines.inpl-nancy.fr

\section{G. VOUILLE}

Expert minier 2, impasse Bel-Air 77300 Fontainebleau gj.vouille@wanadoo.fr
Larticle expose la démarche scientifique élaborée depuis une dizaine d'années dans le bassin minier ferrifère lorrain $\left(1700 \mathrm{~km}^{2}\right)$ pour évaluer les risques d'affaissement progressifs ou d'effondrements brutaux, dans ce contexte géologique et minier particulier et alors que la totalité des exploitations sont aujourd'hui fermées. L'approche repose sur un processus interactif permanent entre d'une part, l'analyse, la compréhension et la modélisation des mécanismes d'instabilités en jeu (défaillance des piliers, rupture du recouvrement) et d'autre part, le retour d'expérience d'une quinzaine de cas bien documentés de phénomènes survenus tout au long du $\mathrm{XX}^{\mathrm{e}}$ siècle. Les paramètres ainsi identifiés comme déterminants à la lumière des mécanismes analysés (résistance ultime des piliers, géométrie des vides abandonnés après exploitation, indices de ( massivité » et de raideur des bancs de recouvrement...) ont fait par ailleurs l'objet d'investigations et de mesures in situ (sur sondages), et en laboratoires (sur échantillons) selon les méthodes classiques de la mécanique des roches.

Le retour d'expérience permet de fixer les valeurs de référence et les seuils pour ces paramètres afin de proposer aux pouvoirs publics une évaluation et une hiérarchisation des risques. Ces éléments fondent les décisions appropriées (surveillance selon différents niveaux d'exigence ou traitement des zones à risques de type expropriation ou comblement) en associant prudence et rigueur.

Mots-clés : mécanique des roches, mines de fer lorraines, après-mines, sécurité.

\section{Lessons learned from experience to ensure security in Lorraine iron mines}

The paper present the scientific approach elaborated over more than 10 years in Lorraine iron basin $\left(1,700 \mathrm{~km}^{2}\right)$ for risk assessment of progressive subsidence or sudden collapses in this particular geological and mining context, and after complete closure of mines.

This approach is founded on a permanent interactive process between, in one hand analysis, understanding and modelling of instabilities (pillar yielding or overburden failures) and, in the other hand, the lessons learnt from experience based upon 15 cases stories, well known and documented phenomena during 20th century.

Parameters considered as determining in regards to the analysed mechanisms (stenght pillars, geometry of abandoned openings, " massivity ) index and stiffness of the overburden strata) have been investigated and measured with classical field and laboratories methods of rock mechanics.

Feed back from experience allows to choice the reference values and thresholds of these parameters in order to propose assessment and hierarchisation of risks to the authorities. These element are the bases for making relievant and rigorous decisions: monitoring with different levels of strictness or treatment for instance expropriation orders or cavities filling.

Key words: rock mechanics, Lorraine iron mines, post-mining, security. 


\section{Présentation du bassin et historique des effondrements}

Le bassin ferrifère lorrain s'étend sur $120 \mathrm{~km}$ entre la frontière luxembourgeoise, au nord, et Neuves-Maisons, au sud de Nancy, et sur $30 \mathrm{~km}$ entre HettangeGrande, à l'est, et Dommary-Baroncourt, à l'ouest. Il est divisé en deux parties principales par l'anticlinal de Pont-à-Mousson : le bassin de Briey-Longwy-Thionville au nord couvrant $1300 \mathrm{~km}^{2}$ et le bassin de Nancy au sud couvrant $380 \mathrm{~km}^{2}$.

\section{1.}

\section{Géologie}

Le gisement ferrifère d'âge Aalénien (150 millions d'années environ) a une épaisseur variable de quelques mètres à une soixantaine de mètres. Il est composé d'une alternance de zones plus riches en fer (30 à $35 \%$ ) et de zones intercalaires. Le minerai se présente sous la forme de petits grains ( $($ oolithes $)$ ), d'oxyde de fer hydraté, de dimensions voisines de quelques dizaines de millimètres et liés entre eux par un ciment constitué essentiellement de calcaire et de silice. Suivant qu'un minerai présente un indice cle basicité $\mathrm{CaO} /$ $\mathrm{SiO}_{2}$ supérieur ou inférieur au seuil de 1,4 correspondant à un mélange auto-fondant dans le haut-fourneau, on désignera le minerai comme "calcaire ) (couches supérieures rouge, jaune, grise) ou ( siliceux ) (couches inférieures brune, noire, verte).

La série ferrifère est surmontée de la série marnocarbonatée du Bajocien qui comprend avec des variations importantes d'épaisseurs et faciès :

- les marnes de Charennes niveau imperméable ;

- une alternance de calcaire et de niveau argileux ou gréseux (calcaires d'Ottange et de Haut-Pont) ;

- les calcaires à polypiers ;

- les calcaires de Jaumont généralement assez massifs.

Le gisement affleure au nord dans la vallée de la Chiers et de l'Alzette et à l'est le long de la vallée de la Moselle. Il s'enfonce avec un pendage de $3 \%$ vers l'ouest pour atteindre une profondeur de $280 \mathrm{~m}$ à la mine d'Amermont-Dommary en limite ouest de la zone exploitable et concédée.

\section{9}

\section{Exploitation}

Du gisement lorrain ont été extraits plus de 3 milliards de tonnes de minerai (1,2 milliard de $\mathrm{m}^{3}$ en volume), la quasi-totalité en un siècle jusqu'en 1997. Les méthodes d'exploitation modernes peuvent être classées en deux catégories selon qu'elles laissent ou non subsister des vides souterrains (Manuel de Mécanique des Roches, tome 2).

Le dépilage intégral consiste à enlever la quasi-totalité du minerai dans un panneau et à faire effondrer le toit immédiat de la couche. Ce foudroyage crée volontairement une cuvette d'affaissement en surface dont les effets débordent de l'aire dépilée au fond selon un angle d'influence.
Lorsque pour protéger la surface (bâtiment, infrastructure) ou pour des raisons économiques le foudroyage est proscrit, des piliers de minerai sont laissés en place pour soutenir le toit. Les panneaux sont découpés par des chambres comprises entre 4 et 7 mètres de large ; les piliers peuvent avoir des formes variées (carrées, rectangulaires, trapézoïdales...). Leur hauteur, égale à l'ouverture exploitée, est comprise entre 3 et 7 mètres et leur largeur varie entre 5 et $25 \mathrm{~m}$. Ces méthodes par chambres et piliers laissent donc subsister des vides après l'arrêt de l'exploitation.

Le problème de la stabilité de tels vides miniers va donc se poser d'abord lors de leur réalisation, puis à long terme au cours du temps ou sous l'influence de modifications de leur environnement. Les difficultés dans la maîtrise de ce problème sont illustrées par l'apparition d’instabilités généralisées aux conséquences parfois meurtrières. Le bassin ferrifère lorrain n'a pas l'exclusivité de ce type de phénomène attaché à la méthode des chambres et piliers abandonnés quelle que soit la substance exploitée; une enquête du Conseil Général des Mines recense en 1965 une soixantaine de cas sur une cinquantaine de sites dont sept cas pour les mines de fer de Lorraine (Chambon et al., 1983). Cellesci, par l'extension de l'emploi de la méthode et du fait des similitudes des conditions naturelles et d'exploitation, constituent donc un bassin de risque important pour la compréhension et la maîtrise des phénomènes d'instabilités généralisées.

\section{3}

\section{Affaissement/effondrements}

Une base de retour d'expérience a été formée avec 16 cas d'instabilités pour lesquels les données retrouvées ont été suffisantes (Tableau I). Dans tous les cas, il s'agit d'un phénomène de grande ampleur affectant plusieurs hectares de chambres et piliers et se traduisant au jour par un affaissement du sol d'ordre métrique.

Les manifestations des événements amènent cependant à différencier deux types de phénomènes se distinguant en particulier par leur cinétique : l'affaissement progressif et l'effondrement brutal.

L'affaissement progressif va se traduire en surface par une cuvette semblable à celle provoquée volontairement par l'opération de dépilage. Au centre de la zone les terrains descendent verticalement; sur les bords internes de la cuvette des manifestations de contraction apparaissent (bourrelets) ; sur les bords externes se manifeste par contre de l'extension (fissures). La cinétique de formation des manifestations en surface est variable d'un cas à l'autre, de l'ordre de la journée pour les plus rapides (Moutiers en 1997, Auboué-Coinville en 1996) à plusieurs mois pour les plus lents (Roncourt en 1999). Après cette phase active les mouvements de tassement se prolongent pendant quelques années comme au-dessus des dépilages.

L'effondrement brutal se traduit au contraire par la descente en bloc de l'ensemble du recouvrement en quelques secondes. Il s'accompagne d'une secousse sismique qui a été enregistrée à Strasbourg et même jusqu'à Moscou pour certains (Roncourt en 1959). La brutalité de l'effondrement des travaux du fond est attestée par le souffle d'air chassé des vides effondrés et dont les effets peuvent être dévastateurs sur l'ensemble de la mine. 


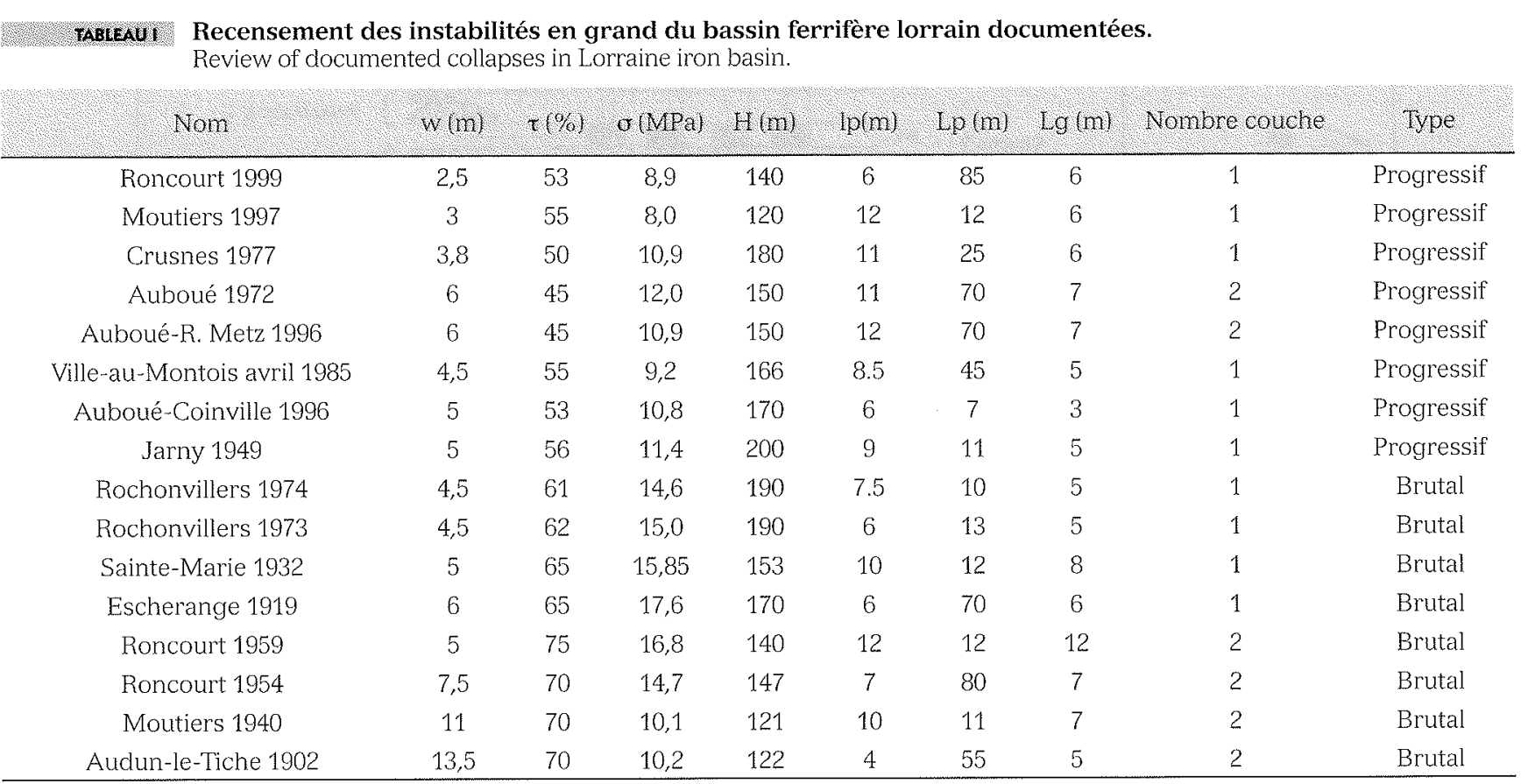

w: ouverture exploitée

$\tau$ : taux de défruitement

$\sigma:$ contrainte sur les piliers

$\mathrm{H}$ : profondeur

Bien que des signes précurseurs aient été la plupart du temps enregistrés durant les jours précédents (craquements, signe de pression, convergence) qui ont permis d'évacuer les quartiers d'exploitation, les chantiers du fond restent accessibles sans dégradations importantes avant le phénomène.

En surface des crevasses ouvertes (jusqu'à $1 \mathrm{~m}$ selon certains témoignages) apparaissent immédiatement sur le pourtour de la zone, le dénivelé de l'affaissement se fait entre les lèvres des crevasses et non selon une cuvette continue.

Le phénomène d'effondrement brutal, du fait de la rapidité de sa mise en place (quelques jours) et de la brutalité de sa manifestation (quelques secondes) présente une dangerosité particulière tant au cours de l'exploitation, qu'après la fermeture de la mine, ce qui justifiera les recherches menées sur le mécanisme de formation et les conditions d'apparition.

Les risques d'affaissement et d'effondrement restent d'actualité même après l'arrêt de l'exploitation des mines; en effet des phénomènes se sont produits alors que les travaux dans la zone avaient cessé depuis de nombreuses années.

\section{2}

\section{Modèle de défaillance des édifices miniers}

\section{1.}

\section{Choix du modèle d'instabilité et chargement des piliers}

Comme rappelé dans le chapitre précédent, la typologie des effondrements miniers dans le bassin ferrifère lorrain au-dessus des zones exploitées par piliers
Ip : largeur pilier

Lp : longueur pilier

Lg: largeur galerie

abandonnés, amène à distinguer les effondrements brutaux, d'une part, et les effondrements progressifs, d'autre part.

Dans tous les cas, même si les mécanismes en jeu varient pour ces différents phénomènes, la géométrie de l'édifice minier souterrain et les propriétés des piliers sont au cœur de leur déclenchement.

L'initialisation et la mise en place du processus d'instabilité commence par la défaillance des piliers, qui se déforment puis se rompent (dans le cas des effondrements brutaux en interaction avec la rupture du recouvrement comme cela sera évoqué au chapitre suivant).

Cette considération, associée au pendage généralement faible (quelques degrés) des couches exploitées et à la grande extension horizontale (pluri-kilométrique) du gisement, a conduit à adopter le modèle simple de l'aire tributaire pour évaluer la charge sur les piliers. Cette méthode de calcul prend directement en compte la profondeur de la zone exploitée et le taux de défruitement. En outre, elle s'adapte bien aux situations de piliers à section irrégulière et au cas des exploitations à couches multiples (Fig. 1).

De plus, la contrainte appliquée sur les piliers, ainsi calculée par le modèle de l'aire tributaire, peut être corrigée selon les configurations d'exploitation situées dans le voisinage de la zone étudiée. Lexemple le plus courant correspond au cas où on se trouve en présence de zones totalement exploitées et foudroyées adjacentes à la zone étudiée. La surcharge introduite est alors de 20 ou $40 \%$ selon la géométrie des travaux.

On dispose ainsi d'un paramètre robuste qui a été calculé sur toutes les zones exploitées partiellement avec piliers abandonnées du bassin ferrifère lorrain soit plusieurs milliers de zones. Létape suivante a consisté à définir l'aléa d'effondrement sur ces zones, en comparant la charge sur les piliers avec leur résistance mécanique. 


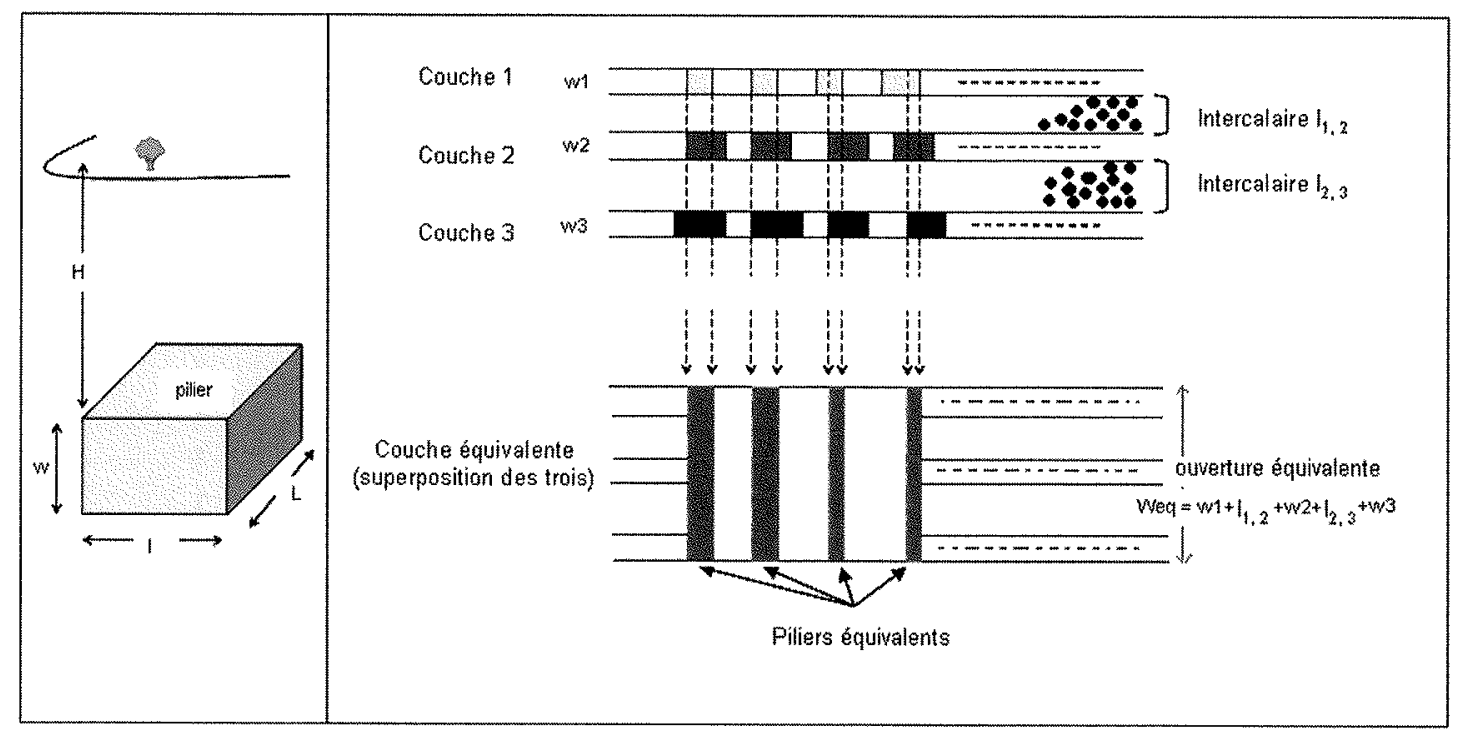

F6. 1 Principaux paramètres utilisés dans le modèle de l'aire tributaire pour une exploitation par chambres et piliers mono ou multicouches, d'après Géodéris.

Main parameters used for tributary area model, in room and pillars mono or multi-seams context, after Géodéris.

Dans le cas particulier des exploitations multicouches, ce paramètre a été complété par l'épaisseur de l'intercalaire séparant deux couches. En première approximation, et au vu de cas d'expérience, il a été admis, d'après l'expérience, qu'une épaisseur d'intercalaire inférieure à $7 \mathrm{~m}$ induisait une instabilité potentielle quelle que soit la charge calculée sur les piliers des différentes couches. La zone correspondante était donc retenue comme zone d'aléa.

\section{9.}

\section{Détermination de la résistance à long terme des piliers}

Pour déterminer la résistance à long terme des piliers, on a classiquement recours à la caractérisation géomécanique à partir d'essais en laboratoire sur échantillons, en prenant en compte toutes les influences connues par la mécanique des roches (effet d'échelle, effet d'élancement, degré de saturation en eau, etc.). Dans le cas présent, ce type de caractérisation a été largement effectué dans le passé en vue d'études particulières de mines ou de quartiers de mines en exploitation ou en projet. La figure 2 rassemble les résultats de ces essais de caractérisations, réalisés par l'École des mines de Paris, sur des éprouvettes cylindriques d'élancement 2 et diamètre $65 \mathrm{~mm}$, selon les méthodes conventionnelles. Une centaine de séries d'essais ont été réunies, chaque point de l'histogramme représentant la moyenne d'une dizaine d'échantillons. Toutefois, étant donné la très grande étendue du gisement et la grande variabilité des propriétés, il est difficile de déduire à partir de ces données une valeur de référence pour la résistance valable pour une étude de risques d'ensemble (sauf à caractériser une à une chaque zone ce qui est évidemment hors de portée).

C'est pourquoi il a été fait appel au retour d'expé140 l'histoire séculaire du bassin. C'est ainsi qu'ont été

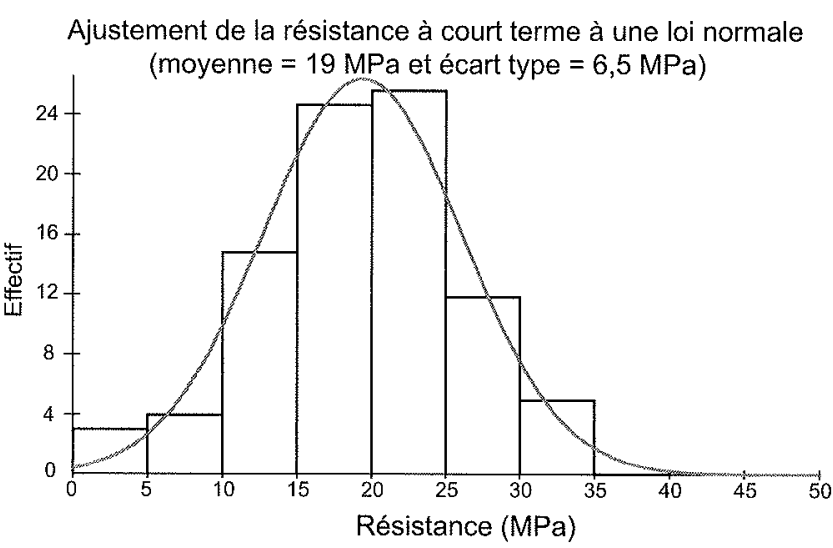

16. 2 Histogramme des résistances moyennes en compression du minerai de fer lorrain d'après toutes les données disponibles. Distribution of mean compressive strenght of Lorraine iron are, compilation of all available data.

analysés les seize cas d'effondrements documentés, survenus au cours du $\mathrm{XX}^{\mathrm{e}}$ siècle et évoqués plus haut. Huit d'entre eux correspondaient à des effondrements brutaux, et huit à des effondrements progressifs. Le tableau I décrit les caractéristiques de ces zones.

On constate que pour tous ces cas d'effondrements avérés, la contrainte sur les piliers calculée par le modèle de l'aire tributaire est systématiquement supérieure ou égale à $8 \mathrm{MPa}$ (cas de Moutiers, en 1997). Pour avoir une marge de sécurité, tenant compte en particulier des imprécisions sur les plans ayant permis le calcul du taux de défruitement, il a été décidé d'adopter la valeur seuill de 7,5 MPa : les zones dont les piliers sont soumis à une charge supérieure ou égale à ce seuil sont considérées comme zones d'aléas d'effondrement et, si des bâtiments ou des infrastructures se trouvent à leur aplomb, sont classées comme zones à risques. Inversement, les zones dont les piliers 
sont sollicités par une charge inférieure à 7,5 MPa sont considérées comme durablement stables, si les conditions d'environnement restent constantes.

Ce résultat appelle quelques commentaires vis-à-vis de la caractérisation présentée dans la figure ci-dessus. En effet, celle-ci fait ressortir une distribution des résistances à la compression simple instantanée ajustable à une loi normale de moyenne $19 \mathrm{MPa}$ et d'écart-type 6,5 $\mathrm{MPa}$. On peut traduire ceci en notant que la valeur seuil de la résistance à long terme, retenue par retour d'expérience, est égale à la valeur moyenne de la résistance à court terme, mesurée sur échantillons en laboratoire, diminuée de près de 1,8 fois l'écart-type, ou encore la valeur seuil (long terme) représente environ $40 \%$ de la résistance moyenne à court terme. Il est intéressant de rapprocher ces valeurs des règles en matière de dimensionnement souvent adoptées dans le passé pour ce type d'exploitation, alors en activité : la valeur de référence préconisée pour la résistance des piliers était égale à la moyenne moins deux écartstypes (pour garantir qu'avec la dispersion d'une distribution gaussienne, moins de 2,5\% des piliers ne risquent une ruine rapide), tandis que la limite de résistance à long terme était estimée de l'ordre de la moitié de la résistånce instantanée. On remarque que malgré des démarches différentes et indépendantes, l'approche par retour d'expérience et l'approche par caractérisation classique fournissent des conclusions très concordantes.

On a par ailleurs vérifié que les zones à risques présentaient des piliers d'élancement inférieur à 1, ce qui ne conduit en aucun cas à corriger à la baisse les valeurs de résistance au motif d'un effet d'élancement.

L'écart entre la charge maximale mesurée en laboratoire et celle qui résulte du retour d'expérience est donc essentiellement imputable pour partie à l'effet d'échelle, à l'influence de l'eau et des marnes du mur, et pour partie à l'effet du vieillissement du minerai de fer qui a été mis en évidence dans des études récentes (Dagallier et al., 2002 ; Grgic et al., 2003).

\section{2.}

\section{Classement des zones d'aléas}

Il a été également possible d'exploiter ces données d'effondrements avec un objectif de sécurité publique. En effet, nous avons souligné l'observation dans le passé de phénomènes brutaux ou au contraire progressifs, ces derniers eux-mêmes se caractérisant par une cinétique plus ou moins rapide. En termes de sécurité publique, il est bien clair que plus les événements sont soudains et se développent rapidement plus il est difficile pour les autorités de prendre les mesures appropriées. D'où l'intérêt d'établir une distinction, parmi les zones à ( aléas effondrements », entre celles menacées d'effondrement brutal ou progressif, et pour ces dernières une classification en fonction de l'importance du riscue encouru, c'est-à-dire de l'association entre l'importance de l'aléa, d'une part, et des conséquences prévisibles en surface au cas où il se réalise, d'autre part.

Le traitement des informations fournies pour les seize cas d'effondrements recensés a été effectué à l'aide d'une méthode statistique d'analyse de données (analyse discriminante), dont le principe et les résultats sont décrits en détail dans le chapitre suivant. Elle permet d'identifier les zones pour lesquelles le risque d'ef- fondrement brutal ne peut être écarté (environ $10 \%$ des zones soit une centaine), et celles qui sont menacées d'un effondrement progressif $(90 \%$ des zones soit près d'un millier).

L'analyse des premières montre qu'il faut faire intervenir un mécanisme d'instabilité complexe mettant enjeu le comportement des terrains du recouvrement absent des facteurs introduits jusqu'à présent dans notre raisonnement (et qui conduira plus loin à la définition d'un critère dit ( géologique )). Leur étude fait l'objet du paragraphe 3 .

Les zones à aléas d'affaissement progressif, quant à elles, peuvent être hiérarchisées en termes de risques, en prenant en compte à la fois la prédisposition d'instabilité de l'édifice minier souterrain, et la quantification des effets à la surface et sa vulnérabilité.

La hiérarchisation s'appuie sur une analyse multicritère, utilisant la méthode Électre TRI. Les critères, qui peuvent être quantitatifs ou qualitatifs, sont pondérés par des coefficients laissés à l'appréciation des utilisateurs, en fonction de l'importance relative qu'ils attribuent à chaque critère. Cette hiérarchisation permet ensuite de définir quelques classes d'action (de surveillance, d'aménagement du sol...) à l'intérieur desquelles chaque zone peut être affectée, au moyen d'une procédure dite d'agrégation. C'est ainsi que les zones à risques sont réparties entre quatre classes de surveillance, allant de la surveillance microsismique continue (classe 1) à un simple nivellement de référence (classe 4).

Les critères de prédisposition à l'instabilité sont : la sollicitation sur les piliers (comme expliqué précédemment) à laquelle s'ajoutent la taille et la forme des piliers, l'ouverture de la couche exploitée, le taux de défruitement, la profondeur, la sensibilité à l'ennoyage (déterminé par la présence de marnes dans les épontes) et les conditions d'environnement du site (présence de failles, d'exploitation voisine...).

Les critères de sensibilité de la surface sont : l'affaissement maximal et la déformation horizontale maximale attendus en cas de rupture, la profondeur, la vulnérabilité des enjeux à la surface (bâti, infrastructures tels que routes, voies ferrées, réseaux, etc.).

Pour la détermination des caractéristiques de la cuvette d'affaissement, un nouveau recours au retour d'expérience a été nécessaire. Il s'est appuyé sur les données recueillies dans 18 cas d'affaissements ou d'effondrements ayant fait l'objet de mesures dans le bassin lorrain (10 cas au-dessus de zones intégralement exploitées et foudroyées soit en couche unique, soit en deux couches, 6 cas correspondant à des effondrements progressifs et 2 à des effondrements brutaux) (Tableau II). Les caractéristiques recherchées sont: l'affaissement maximal Am au centre de la cuvette, la déformation maximale Eh en bordure de cuvette, la pente maximale Pm et l'angle d'influence en bord de cuvette (ces derniers ne sont pas introduits dans l'analyse multicritères).

Ainsi on établit que pour une couche unique, d'ouverture $W$, et une zone de dimension critique ou sur-critique, $\mathrm{Am}=0,5 \times \mathrm{W} \times$ taux de défruitement (1).

Pour plusieurs couches l'affaissement Am est égal à la somme des affaissements induits par chaque couche.

La déformation maximale s'en déduit, pour une profondeur $\mathrm{H}$, par la formule classique $\mathrm{Em}=\propto \times \mathrm{Am} / \mathrm{H}$ (2) et la pente maximale par la formule $\mathrm{Pm}=\beta \times \mathrm{Am} / \mathrm{H}$ (3). 


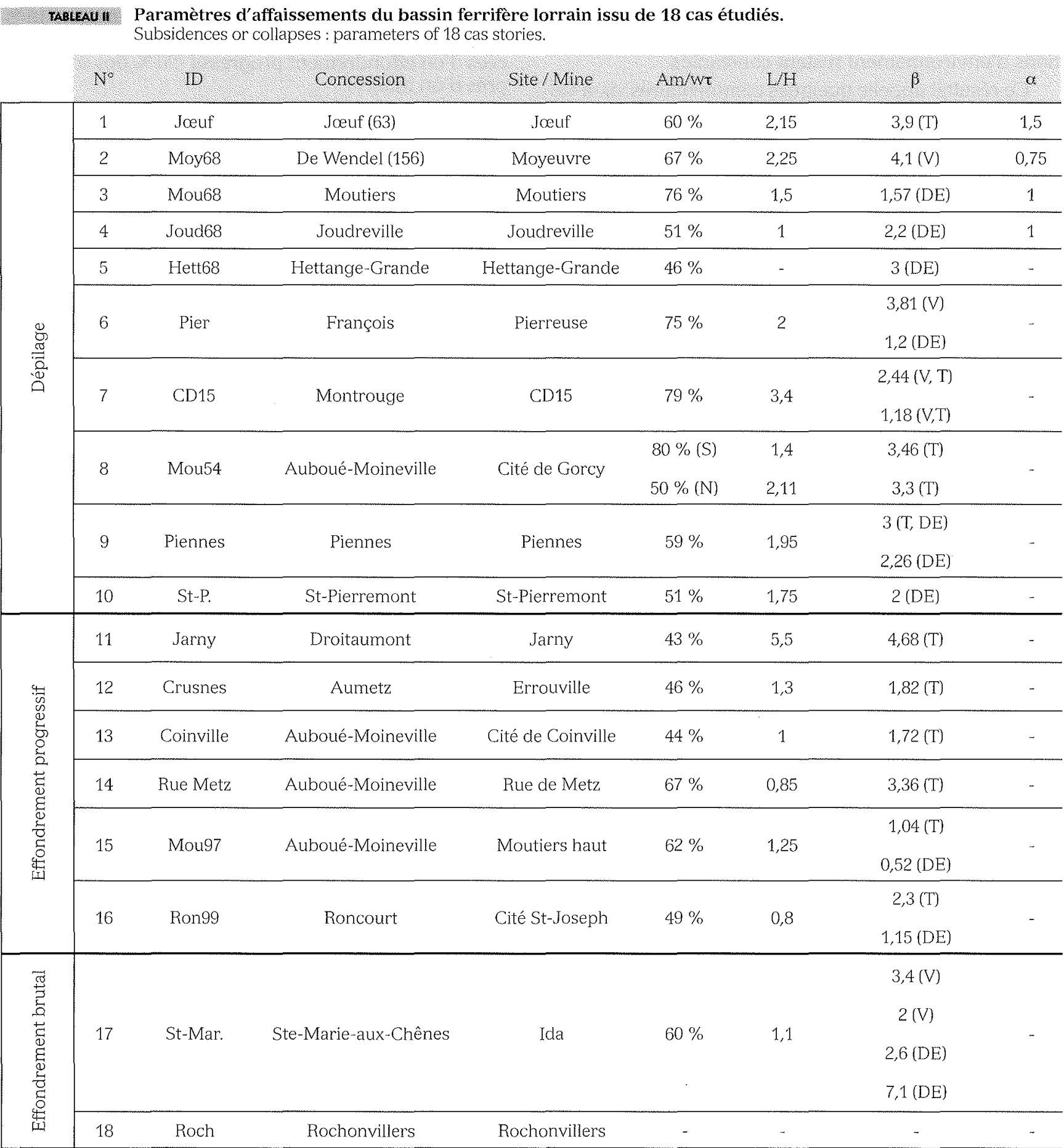

Am : affaissement maximal

w : ouverture exploitée

$\tau$ : taux de défruitement

$\alpha$ et $\beta$ étant deux coefficients déterminés empiriquement.

Le même retour d'expérience a amené à retenir les valeurs : $\alpha=1,5$ et $\beta$ compris entre 3 et 5 selon, respectivement, qu'on se trouve au bord d'une zone voisine vierge (ou faiblement tracée) ou bien exploitée totalement. Ces valeurs sont déterminées pour des déformations exprimées en $\mathrm{mm} / \mathrm{m}$ et des pentes en pourcentage.

L'angle d'influence varie de 10 à $35^{\circ}$ selon respectivement que la zone voisine est vierge ou exploitée totalement $\left(20^{\circ}\right.$ s'il s'agit d'une zone exploitée partielle-
L : largeur du panneau exploité

$\mathrm{H}$ : profondeur

\section{Affaissement/effondrement : le rôle du recouvrement}

Comme nous l'avons déjà indiqué, la reconnaissance des configurations d'exploitation susceptibles de se traduire par un effondrement brutal est particulièrement importante en raison de la rapidité avec laquelle le phénomène se met en place au fond (précurseurs limités à quelques jours avant le phénomène ce qui rend très difficile la mise en place de mesures appropriées de sauvegarde). 
La comparaison des circonstances qui amènent l'occurrence d'un affaissement ou d'un effondrement confirme une différence dans le mécanisme d'évolution de ces deux types d'instabilités, différence proposée par (Tincelin, 1962 et 1982). La recherche consistait alors à définir des critères quantitatifs permettant d'exclure pour une zone d'exploitation la formation d'un effondrement.

\section{3)}

\section{Mécanismes d'instabilité}

Dans le mécanisme de l'affaissement, le phénomène progresse à partir des travaux du fond vers la surface. Au sein d'une zone, les piliers ne sont pas uniformément chargés, certains arriveront plus vite à la rupture du fait de leurs dimensions plus petites, de leur position par rapport à l'environnement de la zone (bord ferme ou dépilage) ou des variations locales de résistance.

La rupture d'un pilier va se traduire par un éboulement du toit immédiat mais pour que le phénomène se propage jusqu'en surface, il faut que la zone affectée au fond soit suffisamment large. En effet aucun affaissement de surface mesurable n'est observé au-dessus d’une zone dépilée dont la largeur est inférieure à $0,4 \mathrm{H}$ (H étant la profondeur de l'exploitation) ; l'affaissement reste très faible jusqu'à $0,6 \mathrm{H}$, puis augmente très rapidement avec la largeur de l'exploitation, ce que l'on peut interpréter comme signe de la rupture du haut toit.

Le processus d'instabilité peut être décrit comme celui de la (c tache » qui s'élargit, la charge que ne supporte plus un pilier rompu étant reportée sur les piliers adjacents, jusqu'à atteindre l'extension permettant la manifestation en surface.

Ce processus d'effet ( dominos ) ne peut pas expliquer l'effondrement instantané de toute la zone pour un matériau aussi peu fragile que le minerai de fer. Pour que tous les piliers arrivent au même instant à la rupture, il faut qu'une sollicitation accrue leur soit imposée à tous au même moment.

Avant l'effondrement, les piliers seraient sollicités au-dessus de leur résistance s'ils avaient à supporter l'ensemble du poids du recouvrement, mais une partie des charges est reprise par le toit qui doit être suffisamment résistant pour assurer ce rôle. C'est ce toit qui a permis la réalisation de ces piliers très sousdimensionnés.

Les contraintes et les déformations vont augmenter dans le toit en cours d'exploitation par augmentation de la largeur du panneau exploité et en post-exploitation par le fluage des piliers. D'autres conditions peuvent remettre en cause l'équilibre toit-pilier : enfoncement dans le mur des piliers, dépilage à proximité de la zone...

Lorsque l'augmentation de la déformation du toit amorce sa rupture et donc la fin de sa contribution à l'équilibre, l'ensemble des piliers est surchargé audelà de leur résistance instantanée ; l'effondrement brutal de l'ensemble des travaux miniers et du recouvrement se produira si la rupture des piliers est de type fragile. Ce sera le cas de petits piliers élancés, fortement chargés, exploités avec un fort taux de défruitement.
Ce mécanisme met en évidence deux conditions nécessaires pour la formation d'un effondrement brutal :

- un banc (ou assemblage de bancs) raide, épais et résistant dans le recouvrement;

- des travaux miniers dans les couches exploitées, très proches de l'instabilité.

Ces deux conditions nécessaires vont permettre de mener l'étude en deux étapes, en définissant d'abord un critère géométrique caractérisant les travaux miniers fragiles dont les données seront obtenues à partir des plans de mine, puis un critère géologique caractérisant les toits raides dont les données obtenues par sondages carottés ne seront à rechercher que sur les zones n'ayant pas satisfait au premier critère.

\section{2}

\section{Critère géométrique représentatif de travaux miniers très fragiles}

Le tableau I montre que les effondrements brutaux sont en moyenne caractérisés effectivement par un niveau de contrainte plus élevé (de l'ordre de $14 \mathrm{MPa}$ contre $10 \mathrm{MPa}$ pour les affaissements progressifs), un élancement ${ }^{1}$ élevé $(1,10$ contre 0,5$)$, une ouverture exploitée importante $(7 \mathrm{~m}$ contre $4,5 \mathrm{~m})$ des piliers peu larges $(7,8 \mathrm{~m}$ contre $9,4 \mathrm{~m})$.

Le taux de défruitement à lui seul permet de discriminer les deux phénomènes; tous les cas d'effondrement ont un taux supérieur à $60 \%$ alors que tous les cas d'affaissement ont un taux inférieur. Il aurait été dangereux de se contenter de ce seul paramètre comme critère ; en effet, l'échantillon de cas n'a pas été constitué a priori de manière à représenter l'ensemble des situations possibles. Les différentes variables ne sont pas indépendantes entre elles, par exemple l'élancement des piliers est très corrélé à l'ouverture cumulée.

Une étude statistique par analyse en composante principale et une étude paramétrique par modélisation des piliers et du recouvrement ont montré que le taux de défruitement limite était susceptible de varier en fonction des autres paramètres, en particulier l'ouverture exploitée (ou l'élancement).

Il est donc préférable de garder d'autres paramètres comme caractéristiques et de rechercher une fonction multivariable permettant de séparer les effondrements des affaissements par la technique de l'analyse discriminante. La meilleure fonction linéaire a été obtenue en combinant les variables : taux de défruitement, contrainte dans les piliers, profondeur, ouverture cumulée, diamètre hydraulique ${ }^{2}$ des piliers, largeur de galerie, coefficient de surcharge. La figure 3 montre que cette fonction permet de distinguer très nettement les huit effondrements brutaux des huit affaissements progressifs. La séparation des deux nuages est suffisamment claire pour qu'il soit possible de fixer une borne ( $f=0,7$ ) permettant d'écarter le risque d'effondrement brutal pour une zone présentant une valeur de la fonction inférieure à cette borne.

\footnotetext{
(1) Dans le cas d'exploitation de deux couches avec un faible intercalaire, l'ouverture et l'élancement sont calculés en considérant l'ensemble des deux couches incluant l'intercalaire.

(2) Le diamètre hydraulique est le rapport entre la surface du pilier et son périmètre, et traduit un « coefficient de forme » du pilier.
} 
La comparaison de la charge sur les piliers dans un modèle d'aire tributaire avec la résistance limite à long terme (voir $\$ 2$ ) a permis de ne retenir sur les 3500 zones (39000 hectares) de chambres et piliers qu'environ. 1000 zones susceptibles de donner lieu à une instabilité en grand; le critère géométrique seul permet d'éliminer le risque d'effondrement brutal sur $90 \%$ de ces zones.

Il reste donc moins de 100 zones (1 600 hectares) pour lesquelles la géologie du toit doit être étudiée.

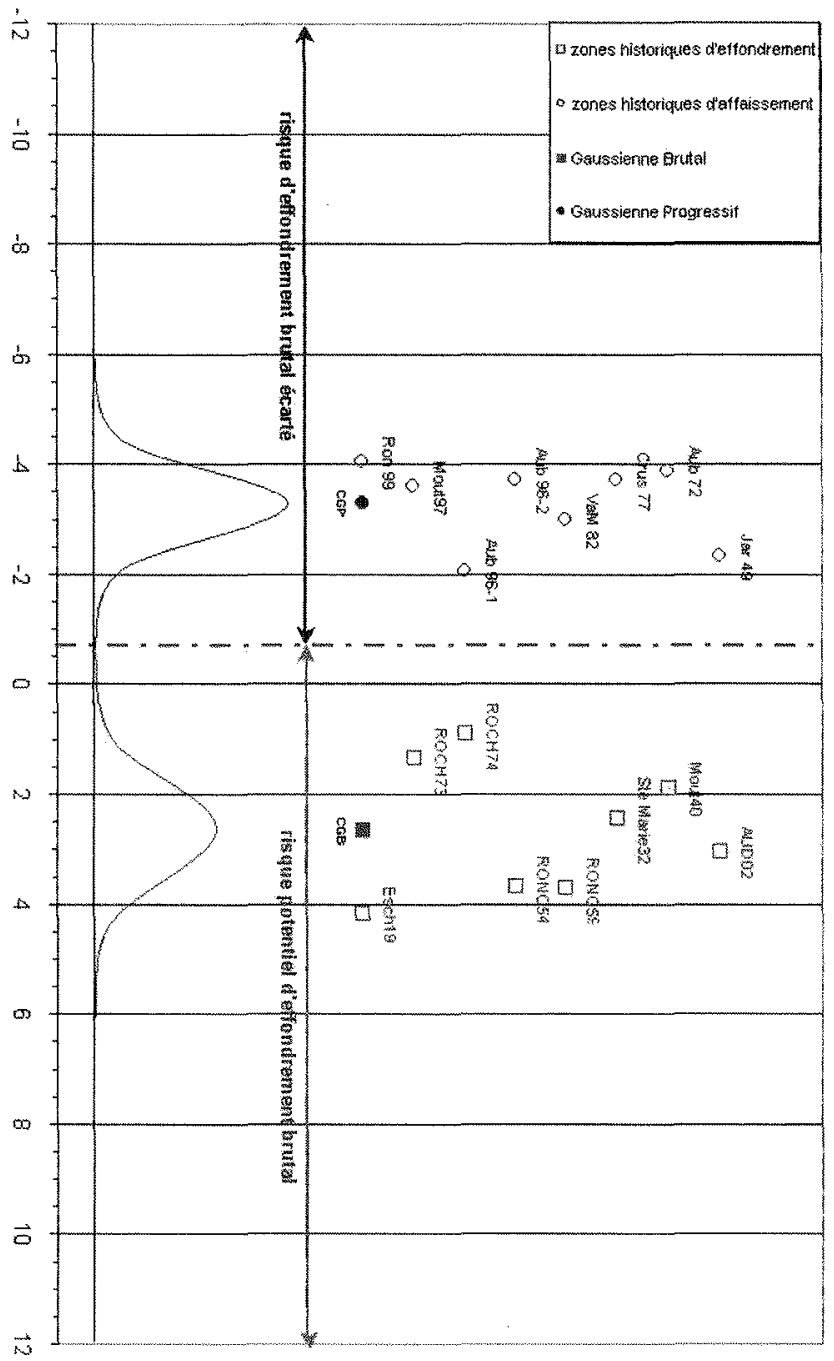

16. 3 Analyse discriminante : affaissements progressifs et effondrements brutaux sur l'axe canonique discriminant, avec critère géométrique.

Discriminating analysis : progressive subsidences and sudden collapses, discriminated with geometrical criteria.

\section{3.}

\section{Critère géologique représentatif d'un recouvrement raide}

Dans le mécanisme de l'effondrement, l'ensemble du recouvrement jouera d'autant mieux son rôle protecteur puis déclencheur qu'il contient des bancs peu déformables et qu'il est donc épais et résistant.

Douze sondages carottés sur l'ensemble du recouvrement (de l'ordre de $150 \mathrm{~m}$ de longueur) ont été réa- lisés à proximité des zones d'instabilités historiques et zones d'aléas les plus importants. Ils ont mis en évidence l'importance des variations verticales comme horizontales de faciès; il n'est pas possible de s'en tenir à la description lithologique classique, un même niveau pouvant contenir des faciès de résistances très différentes (Tableau III).

Un sondage est donc décrit par une succession de bancs de même faciès caractérisés chacun par son épaisseur et un module de déformabilité obtenu en tenant compte des caractéristiques géomécaniques moyennes du faciès et de la fracturation relevée localement sur la carotte (Hoek et Brown, 1997). Deux indices ont été définis à partir de cette caractérisation d'un sondage exprimant :

- la massivité globale de la couverture à partir de la somme des produits des modules de déformabilité multipliée par les épaisseurs des faciès calcaires $A^{\prime}=$ $[\Sigma \mathrm{Em} \times \mathrm{h}] / \mathrm{Emax} . \mathrm{H}(4), \mathrm{H}$ étant la profondeur des travaux ;

- le banc le plus ( raide » $B^{\prime}=\operatorname{Max}(E m$. h)/Emax . hmax.

Pour définir les indices ci-dessus et compte tenu de la variabilité constatée des paramètres, on a retenu pour les coefficients de pondération les valeurs maximales observées quels que soient le faciès et le sondage $(E \max =40 \mathrm{GPa}$ et hmax $=30 \mathrm{~m})(5)$.

$A^{\prime}$ varie entre 0 et $0,5, B^{\prime}$ entre 0 et 1 .

Cette massivité évaluée au niveau d'un sondage doit être complétée pour caractériser le comportement d'un panneau par l'environnement morphologique ; en effet, la morphologie du relief peut favoriser la fracturation verticale et la répartition des contraintes dans le recouvrement. On distinguera ainsi un panneau exploité sous un plateau, de celui exploité sous une pente libre (flanc de vallée) ou encore sous un promontoire (carrefour de vallées).

En combinant ces trois indices et le critère géométrique, un critère global de risque d'effondrement brutal a pu être élaboré ; la figure 4 montre la différenciation des affaissements et des effondrements historiques par ce critère combinant les deux conditions du mécanisme : le toit ( raide ) et les travaux miniers ( fragiles ).

Les zones (une dizaine) sur lesquelles ce risque ne peut être écarté feront l'objet de dispositions spécifiques : surveillance continue sous astreinte par méthode microsismique, en attendant des mesures de mise en sécurité (expropriation, comblement des travaux au fond).

Cette méthodologie a été soumise à examen par un groupe international d'experts en mécanique des Roches (Fairhurst et al., 2003).

\section{Conclusion}

Ce sujet fait toujours l'objet de nouveaux développements. Des travaux sont en cours à Géoderis sur la possibilité de repérer les paramètres capables d'influencer la cinétique des effondrements progressifs, dont on a vu qu'ils étaient susceptibles d'être plus ou moins rapides. Cette question est d'une grande importance pratique, car elle conditionne évidemment les modalités de la surveillance et la rapidité d'intervention en termes de sécurité publique. La réflexion s'oriente notamment vers l'influence possible de la forme des 
Tabteau ui Résistance et module de déformation des faciès de la série sédimentaire.

Uniaxial compressive strenght and Young modulus of different facies in the sedimentary series.

\begin{tabular}{|c|c|c|c|}
\hline Formation & Faciès & $\begin{array}{l}\text { RC } \\
\text { (MPa) }\end{array}$ & $\begin{array}{l}\frac{E}{2} \\
(G \mathrm{~Pa} a \\
\end{array}$ \\
\hline Calcaires de Doncourt & DC : calcaire marneux & 14 & 18 \\
\hline Calcaires de Jaumont & $\begin{array}{l}\mathrm{JM} \text { : calcaire et marne } \\
\mathrm{JCB}: \text { calcaire massif }\end{array}$ & $\begin{array}{l}18 \\
54\end{array}$ & $\begin{array}{l}18 \\
34\end{array}$ \\
\hline Calcaire à polypiers & $\begin{array}{l}\text { PCEAM : calcaire et marne } \\
\text { PCB : calcaire biotherme } \\
\text { PCA : calcarenite poreuse }\end{array}$ & $\begin{array}{l}27 \\
54 \\
26\end{array}$ & $\begin{array}{l}24 \\
44 \\
28\end{array}$ \\
\hline Calcaire de Haut-Pont & $\begin{array}{l}\text { HPC : calcaire blanc } \\
\text { HPG : gréseux }\end{array}$ & $\begin{array}{l}71 \\
17\end{array}$ & $\begin{array}{l}51 \\
12\end{array}$ \\
\hline Calcaire d'Ottange & $\begin{array}{l}\text { OTC : calcaire blanc } \\
\text { OTAG : argileux - gréseux }\end{array}$ & $\frac{22}{7}$ & $\begin{array}{c}20 \\
5\end{array}$ \\
\hline Marne de Charennes & $\begin{array}{l}\text { Marneuse } \\
\text { Carbonatée }\end{array}$ & $\begin{array}{c}9 \\
21\end{array}$ & $\begin{array}{l}5 \\
8\end{array}$ \\
\hline
\end{tabular}

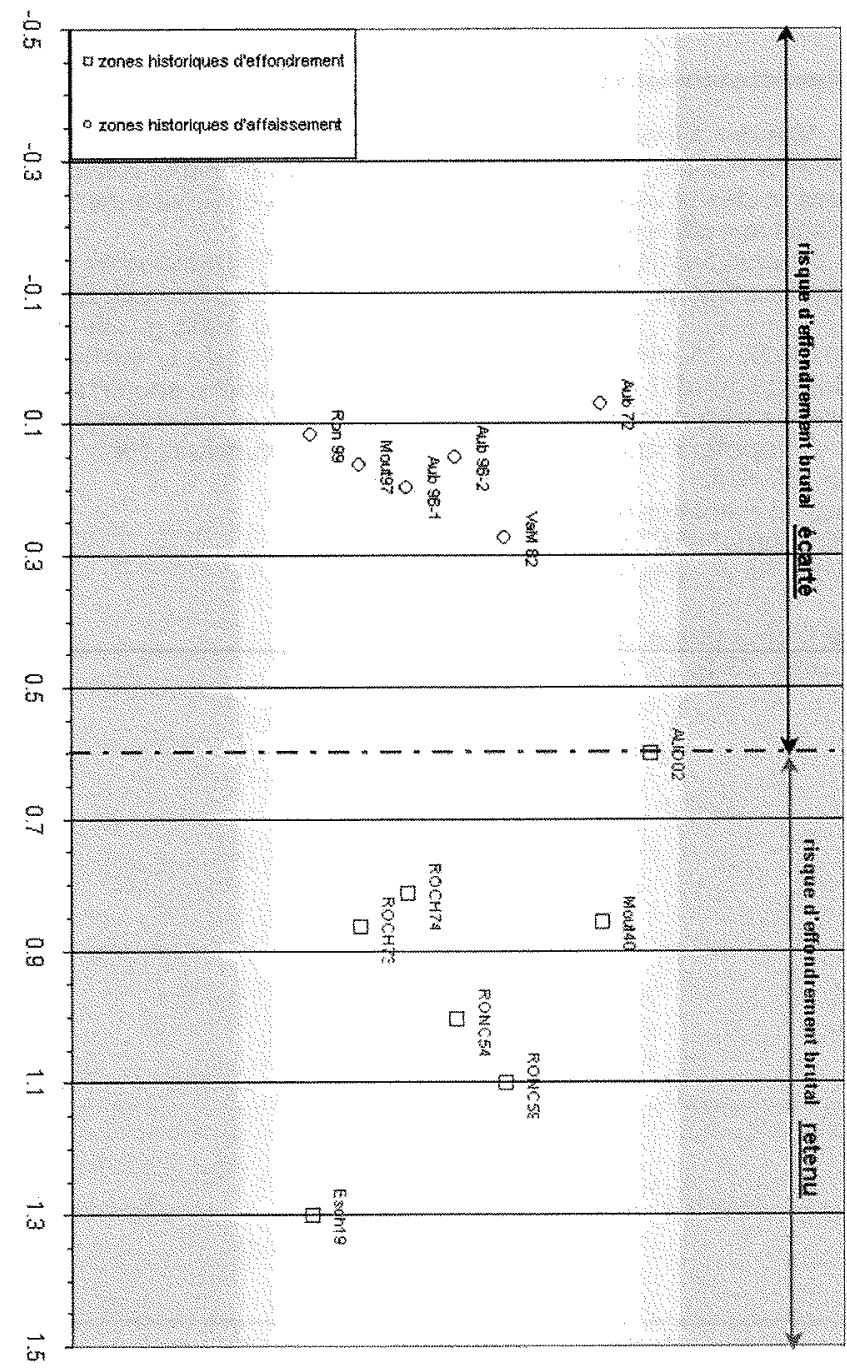

16.
Analyse discriminante : affaissements progressifs et effondrements brutaux avec critère géologique.

Discriminating analysis : progressive subsidence and sudden collapses, discriminated with geological criteria. piliers (plus ou moins allongés) sur la cinématique (plus ou moins lente) de l'affaissement. Un indice de forme et de ( massivité » des piliers discriminant à cet égard est actuellement en cours de mise au point (toujours en regard des comportements réels répertoriés).

Par ailleurs les instabilités potentielles imputables à un intercalaire d'épaisseur inférieure à $7 \mathrm{~m}$, dans le cas des exploitations de couches multiples, sont soumises à un examen détaillé pour les situations dans lesquelles les piliers dans une ou plusieurs couches supportent une charge inférieure au seuil de défaillance à long terme $(7,5 \mathrm{MPa})$. Cet examen ne remet pas en cause l'affectation en zone d'aléa, mais peut modifier le niveau de déformation finale en surface après affaissement, et donc le classement vis-à-vis des risques.

Enfin la question de la vulnérabilité fait l'objet d'une attention particulière avec l'élaboration d'un indicateur amélioré croisant la déformation attendue en surface avec une (c note de vulnérabilité ) qui intègre non seulement la sensibilité du bâti (CSTB) mais prend mieux en compte la densité de population.

Ces développements, destinés à mieux calibrer le niveau d'exigence requis en matière de surveillance, sont tous nourris des données d'expérience recueillies soigneusement depuis plusieurs années par Géoderis dans le bassin ferrifère lorrain.

La conclusion générale qui paraît s'imposer à ce sujet met en lumière l'intérêt d'une démarche coordonnée et interactive entre la compréhension approfondie des mécanismes fondée sur des études théoriques et des modélisations, et l'utilisation des retours d'expériences pour en caler les paramètres et en valider les principes. Le contexte présenté ici illustre spécialement bien ce propos.

L'occurrence des affaissements et effondrements et la nécessité de les prévoir et, si possible, les prévenir a conduit spontanément, dans le domaine minier, à l'élaboration de règles de dimensionnement empiriques, en révision permanente à mesure que de nouveaux cas de figure apparaissent, et, de ce fait, insuffisamment durables pour asseoir solidement une politique de mise en sécurité à long terme. 
Mais, éclairée par la compréhension des mécanismes et leur modélisation avec des calculs validés, l'exploitation des retours d'expérience prend une toute autre dimension. Il devient possible d'identifier les paramètres pertinents et de les évaluer de façon fiable. Réciproquement, l'état des connaissances ainsi atteint autorise à élaborer des modèles opérationnels transposables à des configurations nouvelles.

Ce type d'approche donne confiance pour valider des décisions en matière de sécurité publique. Elle modifierait, le cas échéant, les méthodes de (c design ») d'une nouvelle exploitation dans un contexte comparable, faisant l'économie des périodes d'essais et erreurs pratiquées dans le passé et dont on connaît les conséquences aujourd'hui.

\section{REMGRCIENENTS}

Les résultats présentés dans cet article proviennent pour l'essentiel de travaux d'expertise mis en place et animés pendant plus de dix ans par le Groupement d'intérêt public Géodéris (constitué par le BRGM et l'INERIS pour le compte des pouvoirs publics). Ils s'appuient sur des éléments qui on été rassemblés et structurés par Géodéris.

Parallèlement les questions qui nécessitaient de nouvelles connaissances ont suscité des travaux de recherche cui ont été conduits, en coordination étroite avec l'expertise, dans le cadre du GISOS (Groupement d'intérêt scientifique sur l'impact et la sécurité des ouvraqes souterrains) constitue par des équipes de chercheurs du BRGM, de l'INERIS, des Écoles de géologie et des mines de Nancy (ces dernières au sein de l'Instiiut national polytechnique de Lorrainel et de l'École des mines de Paris.

Que tous les acteurs soient ici remerciés.

\section{Bibliographie}

Chambon C., Arcamone J., Josien J.-P., Piguet J.-P. - Évaluation de la stabilité d'exploitations par chambres et piliers et comportement du toit. $5^{\mathrm{e}}$ Congrès international de mécanique des roches, Melbourne, avril 1983.

CSTB - Typologie du bâti présent dans le bassin ferrifère lorrain. Niveaux d'endommagement (Annexe 10). In Réflexion sur l'évolution de la surveillance microsismique dans le bassin ferrifère lorrain en vue de l'optimisation du dispositif. Rapport Géodéris, 2009.

Dagallier G., Grgic D., Homand F. - Mineralogical and microstructural characterization of the anthropic-origin ageing of iron ore in Lorraine (France). CR Geosciences, 334, 2002, p. 455-462.
Fairhurst C., Piguet J.-P., Van der Merwe N. - Examen de la méthode "Géodéris ») d'évaluation des risques et des conséquences potentielles relatifs aux affaissements de surface dans le bassin ferrifère lorrain. Rapport d'expertise Géodéris 2003.

Grgric D., Homand F., Hoxha D. - A short and long term rheological model to understand the collapses of iron mines in Lorraine (France). Computers and Geotechnics, 30, 2003, p. 557-570.

Hoek E., Brown E.T. - Practical estimates of rock mass strenght. Int. Journal of Rock Mechanics \& Mining Sciences, 34(8) 1997, p. 1165-1186.

Manuel de Mécanique des Roches (tome 2) Les Applications, chapitre 23 (p. 259-
277) et chapitre 26 (p. 319-327). Presses de l'École des mines de Paris, collection Sciences de la Terre et Environnement 2004.

Tincelin E., Sinou P. - Effondrements brutaux et généralisés. Coups de toit. Revue de l'industrie minérale 1962, p. 239-262.

Tincelin E. - La stabilité de la surface à l'aplomb des exploitations souterraines en mines métalliques ou assimilées. Revue de l'industrie minérale, 1982 